\title{
Robotic Assisted, Total Laparoscopic, and Total Abdominal Hysterectomy for Management of Uterine Cancer
}

\author{
Nevadunsky Nicole ${ }^{1}$, Clark Rachel ${ }^{1}$, Muto Michael ${ }^{1,2}$, Berkowitz Ross ${ }^{1,2}$, Ghosh Sue ${ }^{1}$, Vitonis Allison ${ }^{1}$, \\ Feltmate Colleen ${ }^{1,2}$ \\ ${ }^{1}$ Department of Obstetrics and Gynecology, Division of Gynecologic Oncology, Brigham and Women's Hospital, Harvard Medical \\ School, Boston, USA; ${ }^{2}$ Gillette Center for Women's Cancer, Dana-Farber Harvard Cancer Center, Boston, USA. \\ Email:nnevadun@montefiore.org
}

Received February 28, 2012; revised March 29, 2012; accepted April 16, 2012

\begin{abstract}
Objectives: The purpose was to compare robotic assisted total laparoscopic hysterectomy (TRH), laparoscopic assisted hysterectomy (TLH) and total abdominal hysterectomy (TAH) with surgical staging $+/-$ lymphadenectomy for the management of uterine cancer. Methods: Institutional review board approval was obtained and patient characteristics, pathologic data, and data related to the surgical procedure were collected from chart review. Data were analyzed with SAS statistical software. Results: A total of 102 TRHs were compared to $115 \mathrm{TLHs}$ and $79 \mathrm{TAHs}$. There were more grade I and endometrial intraepithelial (EIN) lesions in the preoperative pathology of TLHs $(P<0.01)$. Pelvic lymphadenectomy was performed in $71(70 \%)$ TRH, $46(58 \%)$ TAH, and $28(24 \%)$ TLH cases $(P<0.01)$. Mean surgical time was 203, 133 and 132 minutes for TRHs, TLHs, and TAHs $(P<0.05)$. Estimated blood loss was 69, 86, and 215 $\mathrm{ml}$ for TRH, TLH, and TAH $(P<0.05)$. Blood transfusions were $19 \%$ in TAHs versus $3 \%$ and $2 \%$ in TLHs and TRHs $(P<0.01)$. There were fewer wound infections $(2 \%$ vs. $10 \%)$ in TRHs versus TAHs $(P<0.01)$. Length of stay was shorter for the TRH and TLH groups $(P<0.05)$. Conclusion: Despite longer surgical times, benefits of minimally invasive technology included shortened length of stay, decreased wound infections, transfusions, and blood loss. In our population, procedure selection for TLH versus TRH may have been influenced by lower preoperative grade, with reservation of robotic technology for cases anticipated to be more complex, and therefore justifying increased technology costs and operating times.
\end{abstract}

Keywords: Robotics; Laparoscopy; Endometrial Cancer

\section{Introduction}

Endometrial cancer has been reported to be the most common gynecologic cancer in the United States, with over 40,000 cases diagnosed annually, and over 7000 deaths [1]. The majority of these cancers are diagnosed at early stages with corresponding survival rates of approximately $95 \%$. A comprehensive surgical staging system was recommended by the International Federation of Gynecology and Obstetrics in 1988 [2]. Minimally invasive surgery has since become increasingly accepted amongst gynecologic oncologists for the treatment of endometrial cancer. In 2006, GOG-LAP2 presented randomized clinical data that laparoscopy was feasible for surgical staging of women with stage I-IIA uterine cancer [3]. Advocates of this approach cite decreased morbidity without compromise of disease modifying treatment. Laparoscopic hysterectomy has been associated with improved quality of life, decreased complications, and decreased recovery time when compared to total abdominal hysterectomy [4-6].

Recently several reports have described the application of robotic technology to minimally invasive surgery for endometrial cancer [7-10]. Described advantages of robotic technology include 3-dimensional imaging of the operating field, instruments that move with wrist-like range of motion, lack of fulcrum effect, and steeper learning curve. Retrospective comparisons of robotic assisted hysterectomy (TRH) to total abdominal hysterectomy (TAH) and laparoscopic assisted hysterectomy (TLH) report robotic advantages including decreased length of stay, estimated blood loss, and complication rates [7-10]. However, these initial robotic studies also suggest increased surgical times and unique complications to minimally invasive surgery such as vaginal cuff separation $[9,11]$. Most recently, reports have described cost 
advantages when robotic technology is applied to minimally invasive treatment of uterine cancer [12,13]. The intent of our study was to present a comparison of patient characteristics, histopathological findings, operative outcomes, and complications of patients treated at our institution by TAH, TLH, and TRH.

\section{Materials and Methods}

Institutional review board approval was obtained for retrospective chart review and abstraction of pertinent data. Collected patient information included age, BMI, medical co-morbidities (diabetes, hypertension, obesity, heart disease, kidney disease, and asthma), smoking history, obstetrical history, and previous surgery. Pathological data included preoperative histology and grade, postoperative histology, grade and stage, mean uterine weight, presence of lymph vascular space invasion (LVSI), and positive peritoneal washings. Comparison of operative parameters included length of surgery, estimated blood loss, transfusion rate, length of stay and complications.

All surgeries were performed by one of two gynecologic oncologists with one assistant of various levels of training including, attending level gynecologic oncologist, attending level general gynecologist, gynecologic oncology fellow and third year resident. All TRHs were completed utilizing the da Vinci Surgical System (TRH). All statistical analyses were performed with SAS software. Analysis was performed using ANOVA for continuous and chi-squared tests for discrete variables. $P$-value of $<0.05$ was considered significant.

Standard surgical technique was utilized for TAH, TLH, and TRH. The da Vinci-S system and five trocars placed through the abdominal wall were routinely used for TRHs. Uterine manipulation was facilitated in TLH and TRH cases by a V-CARE uterine manipulator (ConMed, Utica, NY). Decisions regarding the necessity and extent of lymphadenectomy were made by the attending physician based upon intra-operative assessment of frozen section by trained gynecologic pathologists. Standard postoperative care included immediate initiation of postoperative feeding and minimal utilization of intravenous opioids.

\section{Results}

Three hundred and thirteen patients treated for suspected uterine cancer: 110 patients with primary surgical resection attempted by TRH from the inception of our robotics program (August 2006 to January 2009), 123 with primary surgical resection attempted by TLH from the inception of our program for laparoscopic staging for endometrial cancer (June 2005 to January 2009), and 79 patients with primary surgical resection by TAH before minimally invasive techniques were utilized for staging of endometrial cancer at our institution (October 2003 to
June 2005).

Of 110 cases attempted by TRH, eight were converted to open laparotomy, and of 124 cases attempted by TLH, nine cases were converted to open laparotomy. Analyses of the remaining $102 \mathrm{TRH}, 115 \mathrm{TRH}$ and $79 \mathrm{TAH}$ cases were performed for our study.

Patient preoperative characteristics of age, body mass index (BMI), medical co-morbidities and previous abdominal surgery did not differ between groups (Table 1). Obese and morbidly obese patients (BMI > 30) represented $65 \%, 50 \%$, and $55 \%$ of TRH, TLH, and TAH cases respectively.

Patients in the TRH and TLH groups had significantly higher parity and rates of previous vaginal delivery compared to the TAH group, however previous cesarean section history was not different. There was a higher incidence of endometrial intraepithelial (EIN) lesions and grade I lesions in the preoperative pathology specimens of the TLH group $(P<0.05)$ (Table 2$)$.

Postoperative histology, mean uterine weight, lymph vascular space invasion, positive pelvic washings, and stage were similar between all three groups (Table 3). Pelvic lymphadenectomy was performed in $71(70 \%)$ TRH, $46(58 \%)$ TAH, and $28(24 \%)$ TLH cases $(P<0.01)$ Paraaortic lymph node dissection was performed in 6 TAH (8\%), 2 TRH (2\%) and 0 TLH cases. Of those cases in which lymph node dissection was performed, there were no differences in the mean lymph node count between techniques. Mean surgical times for patients who underwent pelvic lymphadenectomy were 210 minutes for TRH, 138 minutes for TAH, and 156 minutes for TLH. There was no significant difference in surgical time between TLH and TRH when surgical procedure included pelvic lymphadenectomy.

First assistants were of attending level in $10 \%$ of robotic and $12 \%$ of laparoscopic cases. Mean surgical time from skin incision to closure was 203 minutes, $133 \mathrm{~min}$ utes and 132 minutes for TRH, TLH, and TAH, respectively $(P<0.05)$ (Table 4$)$. Mean estimated blood loss (EBL) was $49 \mathrm{ml}$ for TRH, $105 \mathrm{ml}$ for TLH, and $150 \mathrm{ml}$ for TAH $(P<0.05)$. Length of hospital stay was significantly shorter for the minimally invasive (TRH and TLH) versus the open TAH group (1.4 days verses 4 days, $P<$ $0.05)$. Blood transfusions were more common in the TAH group (19\%) versus the TLH (3\%) and TRH (3\%) groups $(P<0.001)$ (Table 5). Although complications were similar between groups, there were fewer postoperative wound infections ( $3 \%$ vs. $10 \%$ ) in the TRH group versus the TAH group $(P<0.01)$. Thirty-seven $(36 \%)$ of TRH patients and twenty-nine $(37 \%)$ of TAH patients received adjuvant radiotherapy, in the form of vaginal brachytherapy or external beam pelvic irradiation postoperatively. Postoperative, adjuvant chemotherapy was given to 14 (14\%) of TRH patients and 15 (19\%) of TAH patients. 
Table 1. Comparison of preoperative patient characteristics.

\begin{tabular}{|c|c|c|c|c|}
\hline & TRH & TLH & TAH & $\boldsymbol{P}$ \\
\hline & $\mathrm{n}=102$ & $\mathrm{n}=115$ & $\mathrm{n}=79$ & \\
\hline Median Age (min-max) & $62(35-89)$ & $59(35-81)$ & $62(39-87)$ & \\
\hline Median BMI (min-max) & $33(18-63)$ & $32(19-62)$ & $34(18-61)$ & \\
\hline History Laparotomy & $52(51)$ & $65(57)$ & $39(49)$ & \\
\hline Medical Co-morbidities & $66(68)$ & $77(67)$ & $47(60)$ & \\
\hline Smoker & $6(6)$ & $15(13)$ & $7(9)$ & \\
\hline Previously Smoked & $16(16)$ & $10(13)$ & $10(9)$ & \\
\hline Nullipara & $27(27)$ & $43(54)$ & $27(24)$ & $<0.01$ \\
\hline Vaginal Birth & $65(64)$ & $33(42)$ & $75(65)$ & 0.01 \\
\hline
\end{tabular}

Table 2. Comparison of preoperative histology.

\begin{tabular}{|c|c|c|c|c|}
\hline & TRH & TLH & TAH & $P$ \\
\hline & $\mathrm{n}=102(\%)$ & $\mathrm{n}=115(\%)$ & $\mathrm{n}=79(\%)$ & \\
\hline \multicolumn{5}{|l|}{ Histology } \\
\hline Endometrial Intraepithelial Neoplasia (EIN) & $6(6)$ & $33(29)$ & $11(14)$ & $<0.01$ \\
\hline Endometrioid Adenocarcinoma & $84(83)$ & $71(62)$ & $57(72)$ & \\
\hline Serous Adenocarcinoma & $7(7)$ & $5(4)$ & $3(4)$ & \\
\hline \multicolumn{5}{|l|}{ Grade } \\
\hline 1 & $57(56)$ & $64(56)$ & $34(43)$ & $<0.01$ \\
\hline 2 & $24(24)$ & $7(6)$ & $17(21)$ & \\
\hline 3 & $14(14)$ & $12(10)$ & $14(17)$ & \\
\hline
\end{tabular}

Of the initial 110 attempted robotics procedures, eight were converted to open abdominal procedures and of the 123 attempted laparoscopic procedures, nine were converted to open abdominal procedures for conversion rates of approximately $8 \%$ and $7 \%$ respectively (Table 6). Of robotic conversions three were caused collectively by advanced cancer found at time of surgery, inability to tolerate steep Trendelenberg, and electrocardiogram changes at time of surgery. Eliminating these cases, the technology related conversion rate for robotic cases was only $5 \%$.

\section{Discussion}

Several studies have been presented supporting the use of robotic technology for surgical management of uterine cancer [7-13]. The majority of retrospective reports, including a recent multi-institutional review of 321 patients treated with robotic technology, describe lengthened surgical time, but decreased blood loss, shortened hospital stay and decreased complications [13]. Our length of surgical time required for TRH was significantly longer than for TAH. In agreement with other reports, benefits to our patients of minimally invasive technology (TRH and TLH) included decreased incidence of wound infections, decreased transfusion rates, decreased blood loss and shortened length of stay.

TLH and TAH operative times were not significantly different in our populations, however there were lower preoperative tumor grades in our TLH group. In our population, procedure selection for TLH versus TRH may have been influenced by lower preoperative tumor grade, with reservation of robotic technology for cases that were anticipated to be more complex, and therefore justifying increased technology costs and operating times.

Our initial experience indicates equivalent surgical procedures between TAH and TRH cases. Postoperative adjuvant therapy with radiation and chemotherapy did not differ between our TAH and TRH cases. TLH cases were not included in this comparison as lower postoperative tumor grades may have lead to confounding. Of note, 
Table 3. Comparison of histopathological outcomes.

\begin{tabular}{|c|c|c|c|c|}
\hline & TRH & TLH & TAH & $P$ \\
\hline & $\mathrm{n}=102(\%)$ & $\mathrm{n}=115(\%)$ & $\mathrm{n}=79(\%)$ & \\
\hline \multicolumn{5}{|l|}{ Histology } \\
\hline EIN/No residual carcinoma & $11(11)$ & $23(20)$ & $9(11)$ & \\
\hline Endometrioid Adenocarcinoma & $77(75)$ & $76(66)$ & $57(72)$ & \\
\hline Serous Adenocarcinoma & $7(7)$ & $7(6)$ & $4(5)$ & \\
\hline \multicolumn{5}{|l|}{ Grade } \\
\hline 1 & $46(45)$ & $68(60)$ & $30(40)$ & $<0.01$ \\
\hline 2 & $27(27)$ & $9(8)$ & $21(27)$ & \\
\hline 3 & $17(17)$ & $12(10)$ & $19(24)$ & \\
\hline \multicolumn{5}{|l|}{ Stage } \\
\hline IA-IC & $73(72)$ & $70(62)$ & $51(65)$ & \\
\hline $2 \mathrm{~A}-2 \mathrm{~B}$ & $6(6)$ & $5(4)$ & $6(8)$ & \\
\hline $3 \mathrm{~A}-3 \mathrm{C}$ & $13(13)$ & $11(10)$ & $10(13)$ & \\
\hline Mean Uterine Weight in Grams (min-max) & $131(42-395)$ & $119(20-574)$ & $151(20-942)$ & \\
\hline Pelvic Lymphadenectomy & $71(70)$ & $28(24)$ & $46(58)$ & $<0.01$ \\
\hline Mean Number Pelvic Lymph Nodes (min-max) & $16(2-77)$ & $12(5-23)$ & $12(1-34)$ & \\
\hline Paraaortic Lymphadenectomy & $2(2)$ & $0(0)$ & $6(8)$ & $<0.01$ \\
\hline Present LVSI & $14(14)$ & $11(10)$ & $15(19)$ & \\
\hline Positive Pelvic Washings & $10(10)$ & $13(11)$ & $3(4)$ & \\
\hline
\end{tabular}

Table 4. Comparison of surgical outcomes.

\begin{tabular}{ccccc}
\hline & TRH & TLH & TAH & P \\
\cline { 2 - 4 } & $\mathrm{n}=102$ & $\mathrm{n}=115$ & $\mathrm{n}=79$ & $<0.05$ \\
\hline Mean Surgical Time in Minutes (min-max) & $203(84-396)$ & $133(48-306)$ & $132(51-258)$ & $<0.05$ \\
Mean Surgical Time in Minutes in Patients with & $210(132-396)$ & $138(72-258)$ & $156(111-306)$ & $215(50-2000)$ \\
Lymphadenectomy (min-max) & $69(10-400)$ & $86(10-700)$ & $<0.05$ & $<0.05$ \\
Mean Estimated Blood Loss in mL (min-max) & $1.4(1-9)$ & $1.4(1-9)$ & $3.8)$ & $15)$ \\
Mean Length of Stay (days) (min-max) & &
\end{tabular}

Table 5. Comparison of postoperative complications.

\begin{tabular}{|c|c|c|c|c|}
\hline \multirow{2}{*}{ Complication } & TRH (\%) & TLH (\%) & TAH (\%) & $P$ \\
\hline & $\mathrm{n}=102$ & $\mathrm{n}=115$ & $\mathrm{n}=79$ & \\
\hline Urinary Tract Infection & $4(4)$ & $1(1)$ & $3(4)$ & \\
\hline Vaginal Cuff Separation & $1(1)$ & $1(1)$ & $0(0)$ & \\
\hline Pulmonary Embolism & $0(0)$ & $0(0)$ & $1(1)$ & \\
\hline Cystotomy Repair & $0(0)$ & $0(0)$ & $1(1)$ & \\
\hline Clostridium Difficile Colitis & $0(0)$ & $0(0)$ & $1(1)$ & \\
\hline Postoperative SICU Admission & $0(0)$ & $0(0)$ & $3(4)$ & \\
\hline Postoperative Wound Infection & $2(2)$ & $1(1)$ & $8(10)$ & $<0.01$ \\
\hline Blood Transfusion Given & $3(3)$ & $2(2)$ & $15(19)$ & $<0.01$ \\
\hline
\end{tabular}


Table 6. Cases converted from laparoscopic to open procedures.

\begin{tabular}{ccc}
\hline Conversion Reason & TRH $(\mathrm{n}=7)$ & TLH $(\mathrm{n}=9)$ \\
\hline Adhesions & 2 & 4 \\
Bleeding & 1 & 0 \\
Inability to Manipulate Uterus & 2 & 5 \\
Advanced Carcinoma & 1 & 0 \\
Inability to Tolerate Trendelenberg & 1 & 0 \\
EKG Changes & 1 & 0 \\
\hline
\end{tabular}

minimally invasive technology and utilization of a V-CARE uterine manipulator was not associated with increased incidence of lymph vascular space invasion. There was an increased trend toward positive peritoneal cytology in both the TRH and TLH groups, however it was not significantly different from the TAH group.

Shortcomings of this study are that it was conducted by retrospective chart review and was not randomized or prospective. Additionally, given the long natural disease course, and recurrence times for endometrial cancer it was not possible to comment on overall oncological outcomes such as survival and recurrence.

Robotic assisted technology for surgical treatment of uterine cancer presents several potential benefits for patient quality of life including decreased wound infections, decreased blood transfusions, and shortened hospital stay. In our study similar benefits were seen from application of traditional straight stick laparoscopic technology, however there were significant differences in preoperative stage and grade. In order to derive the optimal benefit of robotic technology, and justify increased operative times and technology costs, it may be useful to continue to evaluate indications and oncological outcomes in women treated for uterine cancer by robotic assisted minimally invasive surgery.

\section{REFERENCES}

[1] A. Jemal, R. Siegel, E. Ward, et al., "Cancer Statistics, 2008," CA: A Cancer Journal for Clinicians, Vol. 58, No. 2, 2008, pp. 71-96. doi:10.3322/CA.2007.0010

[2] J. Mikuta, "International Federation of Gynecology and Obstetrics Staging of Endometrial Cancer," Cancer, Vol. 71, 1988, pp. 1460-1463.

[3] J. Walker, R. Mannel, M. Piedmonte, J. Schlaerth, N. Spirtos and G. Spiegel, "Phase III Trial of Laparoscopy (Scope) vs. TAH (Open) for Surgical Resection and Comprehensive Surgical Staging of Uterine Cancer: A Gyne- cologic Oncology Group (GOG) Study Funded by NCI,' Gynecologic Oncology, Vol. 101, 2006, pp. S11-S12.

[4] K. Hatch, "Laparoscopic Lymphadenectomy and Laparoscopic-Assisted Vaginal Hysterectomy (Editorial)," Gynecologic Oncology, Vol. 190, 2003, pp. 503-504. doi:10.1016/S0090-8258(03)00497-9

[5] S. Ribeiro, R. Ribeiro, N. Santos and J. Pinotti, "A Randomized Study of Total Abdominal, Vaginal, and Laparoscopic Hysterectomy," International Gynecology \& Obstetrics, Vol. 83, No. 1, 2003, pp. 37-43. doi:10.1016/S0020-7292(03)00271-6

[6] Y.-H. Cho, D.-Y. Kim, J.-H. Kim, Y.-M. Kim, Y.-T. Kim and J.-H. Nam, "Laparoscopic Management of Early Uterine Cancer: 10 Year Experience in an Asian Medical Center," Gynecologic Oncology, Vol. 106, No. 3, 2007, pp. 585-590. doi:10.1016/j.ygyno.2007.05.011

[7] J. Boggess, P. Gehrig, L. Cantrell, A. Shafer, M. Ridgeway, E. Skinner and W. Fowler, "A Comparative Study of 3 Surgical Methods for Hysterectomy with Staging for Endometrial Cancer: Robotic Assistance, Laparoscopy, Laparotomy," American Journal of Gynecologic Oncology, Vol. 199, No. 4, 2008, pp. 360.e1-360.e9

[8] S. Denardis, R. Holloway, I. Bigsby, P. Dirk, S. Ahmad and N. Finkler, "Robotically Assisted Laparoscopic Hysterectomy versus Total Abdominal Hysterectomy and Lymphadenectomy for Endometrial Cancer," Gynecologic Oncology, Vol. 111, No. 3, 2008, pp. 412-417. doi:10.1016/j.ygyno.2008.08.025

[9] M. Bell, J. Torgerson, U. Seshadri-Kreaden, A. Wierda Suttle and S. Hunt, "Comparison of Outcomes and Cost for Endometrial Cancer Staging via Traditional Laparotomy, Standard Laparoscopy and Robotic Techniques," Gynecologic Oncology, Vol. 111, No. 3, 2008, pp. 407411. doi:10.1016/j.ygyno.2008.08.022

[10] D. Holtz, G. Miroshnichenko, M. Finnergan and C. Dunton, "Endometrial Cancer Surgery Costs: Da Vinci Robot versus Laparoscopy," Gynecologic Oncology, Vol. 108, Suppl. 1, 2008, p. A322.

[11] L. Seamon, M. Henretta, K. Kim, M. Carlson, D. Cohn and J. Fowler, "Robotic versus Laparoscopic Hysterectomy and Lymphadenectomy for Endometrial Cancer: Conversion Rates and Operating Time," Gynecologic Oncology, Vol. 108, Suppl. 1, 2008, p. A142.

[12] R. Hanna, L. Cantrell, A. Gehrig, J. Mendivil and J. Boggess, "A Cost Analysis of Robotic Surgery as Compared with Conventional Laparoscopy for Endometrial Cancer Staging," Gynecologic Oncology, Vol. 112, Suppl. 1, 2009, p. A330.

[13] M. Lowe, D. Chamberlain, P. Johnson, S. Kamelle and T. Tillmans, "Multi-Institutional Gynecologic Robotic Surgical Consortium: Analysis of Perioperative Outcomes Associated with the Utilization of Robotics in the Surgical Management of Endometrial Cancer," Gynecologic Oncology, Vol. 112, Suppl. 1, 2009, p. A333. 